\title{
Global Asymptotic Stability of a Nonautonomous Difference Equation
}

\author{
Mehmet Gümüşs ${ }^{1}$ and Özkan Öcalan ${ }^{2}$ \\ ${ }^{1}$ Department of Mathematics, Faculty of Science and Arts, Bülent Ecevit University, 67100 Zonguldak, Turkey \\ ${ }^{2}$ Department of Mathematics, Faculty of Science and Arts, Afyon Kocatepe University, ANS Campus, 03200 Afyonkarahisar, Turkey
}

Correspondence should be addressed to Mehmet Gümüş; m.gumus@karaelmas.edu.tr

Received 16 May 2014; Revised 21 July 2014; Accepted 21 July 2014; Published 11 August 2014

Academic Editor: Chein-Shan Liu

Copyright ( 2014 M. Gümüş and Ö. Öcalan. This is an open access article distributed under the Creative Commons Attribution License, which permits unrestricted use, distribution, and reproduction in any medium, provided the original work is properly cited.

We study the following nonautonomous difference equation: $x_{n+1}=\left(x_{n} x_{n-1}+p_{n}\right) /\left(x_{n}+x_{n-1}\right), n=0,1, \ldots$, where $p_{n}>0$ is a period-2 sequence and the initial values $x_{-1}, x_{0} \in(0, \infty)$. We show that the unique prime period-2 solution of the equation above is globally asymptotically stable.

\section{Introduction}

In [1], Xianyi and Deming studied the global asymptotic stability of positive solutions of the difference equation

$$
x_{n+1}=\frac{x_{n} x_{n-1}+p}{x_{n}+x_{n-1}}, \quad n=0,1, \ldots,
$$

where $p \in[0, \infty)$ and the initial conditions are positive real numbers.

The mathematical modeling of a physical, physiological, or economical problem very often leads to difference equations (for partial review of the theory of difference equations and their applications). Moreover, a lot of difference equations with periodic coefficients have been applied in mathematical models in biology. In addition, between others in [2-10], we can see some more difference equations with periodic coefficient that have been studied. For constant coefficient, see $[1,11-15]$ and the references cited therein.

In the present paper, we consider the following nonautonomous difference equation:

$$
x_{n+1}=\frac{x_{n} x_{n-1}+p_{n}}{x_{n}+x_{n-1}}, \quad n=0,1, \ldots
$$

where $\left\{p_{n}\right\}$ is a two periodic sequence of nonnegative real numbers and the initial conditions $x_{-1}, x_{0}$ are arbitrary positive numbers.

The autonomous case of (2) is (1).

It is natural problem to investigate the behavior of the solutions of (1) where we replace the constant $p$ by a nonnegative periodic sequence $p_{n}$. That is, we will consider the recursive sequence (2) where $\left\{p_{n}\right\}$ is a two-periodic sequence of nonnegative real numbers. What do the solutions of (2) with positive initial conditions $x_{-1}$ and $x_{0}$ do?

Our aim in this paper is to investigate the question above and extend some results obtained in [1]. More precisely, we study the existence of a unique positive periodic solution of (2) and we investigate the boundedness character and the convergence of the positive solutions to the unique two periodic solution of (2). Finally, we study the global asyptotic stability of the positive periodic solution (2).

As far as we examine, there is no paper dealing with (2). Therefore, in this paper, we focus on (2) in order to fill the gap.

Now, assume that

$$
p_{n}= \begin{cases}\alpha & \text { if } n \text { is even } \\ \beta & \text { if } n \text { is odd }\end{cases}
$$


in (2). Then,

$$
\begin{array}{ll}
x_{2 n+1}=\frac{x_{2 n} x_{2 n-1}+\alpha}{x_{2 n}+x_{2 n-1}}, & n=0,1, \ldots, \\
x_{2 n+2}=\frac{x_{2 n+1} x_{2 n}+\beta}{x_{2 n+1}+x_{2 n}}, & n=0,1, \ldots,
\end{array}
$$

where $x_{-1}, x_{0} \in(0, \infty)$.

Here, we review some results which will be useful in our investigation of the behavior of positive solutions of (2).

Let $I$ be some interval of real numbers and let

$$
f: I \times I \longrightarrow I
$$

be a continuously differentiable function. Then for every pair of initial values $x_{-1}, x_{0} \in I$, the difference equation

$$
x_{n+1}=f\left(x_{n}, x_{n-1}\right), \quad n=0,1, \ldots,
$$

has a unique solution $\left\{x_{n}\right\}$.

Definition 1 . Let $(\bar{x}, \bar{y})$ be an equilibrium point of the map

$$
B=\left(f, u_{n}, u_{n-1}, \ldots, u_{n-k}, g, z_{n}, \ldots, z_{n-k}\right),
$$

where $f$ and $g$ are continuously differentiable functions at $(\bar{x}, \bar{y})$. The linearized system of

$$
\begin{aligned}
& u_{n+1}=f\left(u_{n}, u_{n-1}, \ldots, u_{n-k}, z_{n}, \ldots, z_{n-k}\right), \\
& z_{n+1}=g\left(u_{n}, u_{n-1}, \ldots, u_{n-k}, z_{n}, \ldots, z_{n-k}\right)
\end{aligned}
$$

about the equilibrium point $(\bar{x}, \bar{y})$ is $v_{n+1}=B\left(v_{n}\right)=B_{j} v_{n}$, where

$$
v_{n}=\left(\begin{array}{c}
u_{n} \\
\vdots \\
u_{n-k} \\
v_{n} \\
\vdots \\
v_{n-k}
\end{array}\right)
$$

and $B_{j}$ is the Jacobian matrix of system (8) about the equilibrium point $(\bar{x}, \bar{y})$.

Theorem 2 (linearized stability). Consider the following.

(1) If both roots of the quadratic equation

$$
\lambda^{2}-p \lambda-q=0
$$

lie in the open unit disk $|\lambda|<1$, then the equilibrium $\bar{x}$ of (6) is locally asymptotically stable.

(2) If at least one of the roots of (10) has absolute value greater than one, then the equilibrium $\bar{x}$ of (6) is unstable.

(3) A necessary and sufficent condition for both roots of (10) to lie in the open unit disk $|\lambda|<1$ is

$$
|p|<1-q<2 \text {. }
$$

(4) A necessary and sufficent condition for both roots of (10) to have absolute value greater than one is

$$
|q|>1, \quad|p|<|1-q| \text {. }
$$

(5) A necessary and sufficent condition for one root of (10) to have absolute value greater than one and for the other to have absolute value less than one is

$$
p^{2}+4 q>0, \quad|p|>|1-q| \text {. }
$$

(6) A necessary and sufficent condition for a root of (10) to have absolute value equal to one is

$$
|p|=|1-q|
$$

or

$$
q=-1, \quad|p| \leq 2 .
$$

\section{Boundedness of Solutions to (2)}

In this section, we mainly investigate the boundedness character of (2), assuming (3) is satisfied.

Theorem 3. Let $\alpha, \beta>0$. Then, every positive solution of (2) is bounded.

Proof. Suppose for the sake of contadiction that $\left\{x_{n}\right\}$ is an unbounded solution of (2). Then, there exist the subsequences $\left\{x_{2 n+1}\right\}$ and $\left\{x_{2 n+2}\right\}$ of the solution $\left\{x_{n}\right\}$ such that one of the following statements is true.

(i) $x_{2 n+1} \rightarrow \infty$ and $x_{2 n+2} \rightarrow a$ ( $a$ is arbitrary positive real number) for all $n \geq 0$.

(ii) $x_{2 n+1} \rightarrow b$ (b is arbitrary positive real number) and $x_{2 n+2} \rightarrow \infty$ for all $n \geq 0$.

(iii) $x_{2 n+1} \rightarrow \infty$ and $x_{2 n+2} \rightarrow \infty$ for all $n \geq 1$.

Now, we will show that the above statements are never satisfied.

From (2), we have the following inequalities:

$$
\begin{aligned}
& x_{2 n+1}<x_{2 n-1}+\frac{\alpha}{x_{2 n}}, \quad \text { for } n \geq 0, \\
& x_{2 n+1}<x_{2 n}+\frac{\alpha}{x_{2 n-1}}, \quad \text { for } n \geq 0, \\
& x_{2 n+2}<x_{2 n}+\frac{\beta}{x_{2 n+1}}, \text { for } n \geq 0, \\
& x_{2 n+2}<x_{2 n+1}+\frac{\beta}{x_{2 n}}, \quad \text { for } n \geq 0 .
\end{aligned}
$$

If we get limits on both sides of the above inequalities, they contradict cases (i) and (ii). From (16) and using the induction, we obtain that

$$
\begin{aligned}
& x_{2 n+1}<x_{-1}+\alpha\left(\frac{1}{x_{0}}+\cdots+\frac{1}{x_{2 n}}\right), \\
& x_{2 n+2}<x_{0}+\beta\left(\frac{1}{x_{1}}+\cdots+\frac{1}{x_{2 n+1}}\right) .
\end{aligned}
$$


Also, if we consider the limiting case of the above inequalities, they contradict case (iii) and complete the proof.

\section{Periodicity and Stability of Solutions to (2)}

In this section, we show that (2) has the unique two-periodic solution and the unique prime period- 2 solution of this equation is locally asymptotically stable.

Theorem 4. Suppose that $p_{n}>0$ is a two periodic sequence such that

$$
p_{n+2}=p_{n}, \quad n=0,1, \ldots
$$

Then (2) has the unique two-periodic solution.

Proof. Let $x_{n}$ be a solution of (2). Using (18), $x_{n}$ is twoperiodic solution if and only if $x_{-1}$ and $x_{0}$ satisfy

$$
x_{-1}=x_{1}=\frac{x_{0} x_{-1}+\alpha}{x_{0}+x_{-1}}, \quad x_{0}=x_{2}=\frac{x_{0} x_{1}+\beta}{x_{0}+x_{1}} \text {. }
$$

We set $x_{-1}=x$ and $x_{0}=y$; then from (19) we obtain the system of equations

$$
x=\frac{x y+\alpha}{x+y}, \quad y=\frac{x y+\beta}{x+y} .
$$

We prove that (2) has a solution $(\bar{x}, \bar{y}), \bar{x}, \bar{y}>0$. From (20) we get

$$
x^{2}+x y=x y+\alpha, \quad y^{2}+x y=x y+\beta
$$

It follows that (2) has a prime period-2 solution if and only if $\alpha, \beta \in(0, \infty), \alpha \neq \beta$, in which case (2) has a unique twoperiodic solution

$$
\sqrt{\alpha}, \sqrt{\beta}, \ldots, \sqrt{\alpha}, \sqrt{\beta}, \ldots
$$

Theorem 5. Assume that $p_{n}>0$ is a two periodic sequence and

$$
\alpha, \beta \in(0, \infty)
$$

Then (2) has the unique prime period-2 solution which is locally asymptotically stable.

Proof. Let $x_{n}$ be a positive solution of (2) and let

$$
\ldots, \sqrt{\alpha}, \sqrt{\beta}, \sqrt{\alpha}, \sqrt{\beta}, \ldots, \sqrt{\alpha}, \sqrt{\beta}, \ldots
$$

be the unique prime period-2 solution of (2). For any $n \geq 0$, set

$$
u_{n}=x_{2 n-1}, \quad v_{n}=x_{2 n} .
$$

Then, (2) is equivalent to the system

$$
u_{n+1}=\frac{u_{n} v_{n}+\alpha}{u_{n}+v_{n}}, \quad v_{n+1}=\frac{u_{n}\left(v_{n}^{2}+\beta\right)+(\alpha+\beta) v_{n}}{v_{n}\left(2 u_{n}+v_{n}\right)+\alpha} \text {. }
$$

So, in order to study (2) we investigate system (26).

Let $H$ be the function on $(0, \infty)^{2}$ defined by

$$
H\left(\begin{array}{l}
u \\
v
\end{array}\right)=\left(\begin{array}{c}
\frac{u v+\alpha}{u+v} \\
\frac{u\left(v^{2}+\beta\right)+(\alpha+\beta) v}{v(2 u+v)+\alpha}
\end{array}\right)=\left(\begin{array}{l}
g(u, v) \\
h(u, v)
\end{array}\right) .
$$

Then,

$$
\left(\begin{array}{l}
\sqrt{\alpha} \\
\sqrt{\beta}
\end{array}\right)
$$

is a fixed point of $H$. Clearly the unique prime period-2 solution is locally asymptotically stable when the eigenvalues of the jakobian matrix $J_{H}$, evaluated at $\left(\begin{array}{c}\sqrt{\alpha} \\ \sqrt{\beta}\end{array}\right)$, lie inside the unit disk. We have

$$
J_{H}\left(\begin{array}{l}
\sqrt{\alpha} \\
\sqrt{\beta}
\end{array}\right)=\left(\begin{array}{ll}
\frac{\partial g}{\partial u}(\sqrt{\alpha}, \sqrt{\beta}) & \frac{\partial g}{\partial v}(\sqrt{\alpha}, \sqrt{\beta}) \\
\frac{\partial h}{\partial u}(\sqrt{\alpha}, \sqrt{\beta}) & \frac{\partial h}{\partial v}(\sqrt{\alpha}, \sqrt{\beta})
\end{array}\right) .
$$

The linearized system of $(26)$ about $(\sqrt{\alpha}, \sqrt{\beta})$ is the system

$$
z_{n+1}=B z_{n}
$$

where

$$
B=\left(\begin{array}{cc}
\frac{\beta-\alpha}{\alpha+\beta+2 \sqrt{\alpha \beta}} & 0 \\
0 & \frac{\alpha-\beta}{\alpha+\beta+2 \sqrt{\alpha \beta}}
\end{array}\right), \quad z_{n}=\left(\begin{array}{c}
u_{n} \\
v_{n}
\end{array}\right) .
$$

The characteristic equation of $B$ is

$$
\begin{gathered}
\lambda^{2}-\left(\frac{\beta-\alpha}{\alpha+\beta+2 \sqrt{\alpha \beta}}+\frac{\alpha-\beta}{\alpha+\beta+2 \sqrt{\alpha \beta}}\right) \lambda \\
-\frac{(\alpha-\beta)^{2}}{(\alpha+\beta+2 \sqrt{\alpha \beta})^{2}}=0
\end{gathered}
$$

and thus

$$
\lambda^{2}-\frac{(\alpha-\beta)^{2}}{(\alpha+\beta+2 \sqrt{\alpha \beta})^{2}}=0 .
$$

From Theorem 2 and (23) all the roots of (33) are of modulus less than 1 . So the system (26) is locally asymptotically stable. Therefore, (2) has the unique prime period-2 solution which is locally asymptotically stable. 


\section{Global Asymptotic Stability for (2)}

In this section, we give our main result. Before doing so, in the next theorem, we will provide an alternative proof for the global stability of (1). In the proof of the main theorem, we will use a similar technique.

Theorem 6. The positive equilibrium point $\bar{x}$ of (1) is globally asymptotically stable.

Proof. We know from Theorem 3 that every positive solution of (1) is bounded for $p \in(0, \infty)$ and we know from [1] that the positive equilibrium point $\bar{x}$ of (1) is locally asymptotically stable for $p \in(0, \infty)$.

Let $\left\{x_{n}\right\}$ be a positive solution of (1) and set

$$
\lim \sup x_{n}=K, \quad \liminf x_{n}=k .
$$

Then by the boundedness of $\left\{x_{n}\right\} ; k, K$ are positive real numbers.

Thus, it is easy to see from (1) that

$$
\begin{gathered}
k \geq \frac{k^{2}+p}{2 K}, \quad K \leq \frac{K^{2}+p}{2 k}, \\
k^{2}+p \leq 2 K k \leq K^{2}+p .
\end{gathered}
$$

Thus, we have

$$
k^{2} \leq K^{2}
$$

and so

$$
k \leq K
$$

From (37), if $k=K$, the proof is obvious. If $k<K$, this case contradicts the unique equilibrium solution of (1) which is locally asymptotically stable. Consequently, it must be $K=k$. This completes the proof.

Now, we are ready to give the proof of the main result.

Theorem 7. Assume that $p_{n}>0$ is a two-periodic sequence and $\alpha, \beta \in(0, \infty)$; then (2) has the unique prime period- 2 solution which is globally asymptotically stable.

Proof. We must prove that the unique prime period-2 solution of (2) is both locally asymptotically stable and globally attractive. We know from Theorem 5 that the unique prime period-2 solution of (2) is locally asymptotically stable for $\alpha, \beta \in(0, \infty)$. It remains to verify that every positive solution of (2) converges the unique prime period-2 solution. Since $\alpha, \beta \in(0, \infty)$, by Theorem 3 every positive solution of (2) is bounded. Let $\left\{x_{n}\right\}$ be a positive solution of (2) and set

$$
\begin{array}{ll}
\lim \sup x_{2 n+1}=M, & \limsup x_{2 n}=S, \\
\liminf x_{2 n+1}=m, & \liminf x_{2 n}=s .
\end{array}
$$

Then by the boundedness of $\left\{x_{n}\right\}, m, M, s$, and $S$ are positive real numbers. Moreover, it is obvious that since $\alpha \neq \beta$, then from (2)

$$
\lim _{n \rightarrow \infty} x_{2 n} \neq \lim x_{2 n+1},
$$

and it is clear that if $\lim x_{2 n+1}$ exists, then so does $\lim _{n \rightarrow \infty} x_{2 n}$. Thus, it is easy to see from (2) that

$$
\begin{array}{ll}
m \geq \frac{m s+\alpha}{M+S}, & M \leq \frac{M S+\alpha}{m+s}, \\
s \geq \frac{m s+\beta}{M+S}, & S \leq \frac{M S+\beta}{m+s} .
\end{array}
$$

From (40), we have

$$
\begin{gathered}
m M+m S-m s \geq \alpha \geq m M+s M-M S \\
m M+m S-m s \geq m M+s M-M S \\
S \geq s .
\end{gathered}
$$

From (41), we have

$$
\begin{gathered}
s M+s S-m s \geq \beta \geq m S+s S-M S, \\
s M+s S-m s \geq m S+s S-M S, \\
M \geq m .
\end{gathered}
$$

With cases (42) and (43), if $s=S$ and $m=M$, the proof is obvious. If $s<S$ and $m<M$, this case contradicts the unique prime period-2 solution of (2) which is locally asymptotically stable. Consequently, it must be $s=S$ and $M=m$. The proof is complete.

\section{Conflict of Interests}

The authors declare that there is no conflict of interests regarding the publication of this paper.

\section{Acknowledgments}

The authors would like to thank the referees for their helpful suggestions. This research is supported by TUBITAK and Bulent Ecevit University Research Project Coordinatorship.

\section{References}

[1] L. Xianyi and Z. Deming, "Global asymptotic stability in a rational equation," Journal of Difference Equations and Applications, vol. 9, no. 9, pp. 833-839, 2003.

[2] R. Devault, V. L. Kocic, and D. Stutson, "Global behavior of solutions of the nonlinear difference equation $x_{n+1}=p_{n}+x_{n-1} / x_{n}$," Journal of Difference Equations and Application, vol. 11, no. 8, pp. 707-719, 2005.

[3] E. A. Grove and G. Ladas, Periodicities in Nonlinear Difference Equations, Chapman and Hall/CRC Press, 2005.

[4] M. R. S. Kulenović, G. Ladas, and C. B. Overdeep, " On the dynamics of $x_{n+1}=p_{n}+\left(x_{n-1} / x_{n}\right)$," Journal of Difference Equations and Applications, vol. 9, no. 11, pp. 1053-1056, 2003. 
[5] M. R. C. Kulenović, G. Ladas, and C. B. Overdeep, "On the dynamics of $x_{n+1}=p_{n}+\left(x_{n-1} / x_{n}\right)$ with a period-two coefficient," Journal of Difference Equations and Applications, vol. 10, no. 10, pp. 905-914, 2004.

[6] V. G. Papanicolaou, "On the asymptotic stability of a class of linear difference equations," Mathematics Magazine, vol. 69, no. 1, pp. 34-43, 1996.

[7] G. Papaschinopoulos, C. J. Schinas, and G. Stefanidou, "On a difference equation with 3-periodic coefficient," Journal of Difference Equations and Applications, vol. 11, no. 15, pp. 12811287, 2005.

[8] G. Papaschinopoulos and C. J. Schinas, "On a $(k+1)$-order difference equation with a coefficient of period $k+1$," Journal of Difference Equations and Applications, vol. 11, no. 3, pp. 215-225, 2005.

[9] Ö. Öcalan, "Asymptotic behavior of a higher-order recursive sequence," International Journal of Difference Equations, vol. 7, no. 2, pp. 175-180, 2012.

[10] Ö. Öcalan, "Dynamics of the difference equation $x_{n+1}=p_{n}+$ $x_{n-k} / x_{n}$ with a period-two coefficient," Applied Mathematics and Computation, vol. 228, pp. 31-37, 2014.

[11] E. M. Elabbasy, H. A. El-Metwally, and E. M. Elsayed, "Global behavior of the solutions of some difference equations," Advances in Difference Equations, vol. 2011, article 28, 2011.

[12] E. M. Elsayed and B. D. Iričanin, "On a max-type and a min-type difference equation," Applied Mathematics and Computation, vol. 215, no. 2, pp. 608-614, 2009.

[13] E. M. Elsayed, "Solutions of rational difference systems of order two," Mathematical and Computer Modelling, vol. 55, no. 3-4, pp. 378-384, 2012.

[14] V. L. Kocic and G. Ladas, Global Behavior of Nonlinear Difference Equations of Higher Order with Applications, vol. 256 of Mathematics and its Applications, Kluwer Academic, Dordrecht, The Netherlands, 1993.

[15] M. R. S. Kulenović and G. Ladas, Dynamics of Second Order Rational Difference Equations: With Open Problems and Conjectures, Chapman \& Hall/CRC, 2001. 


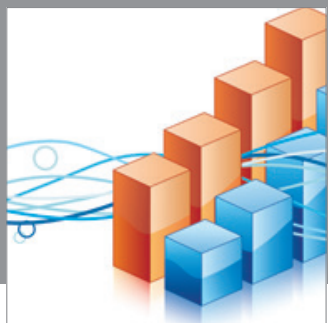

Advances in

Operations Research

mansans

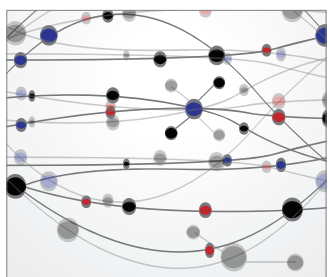

The Scientific World Journal
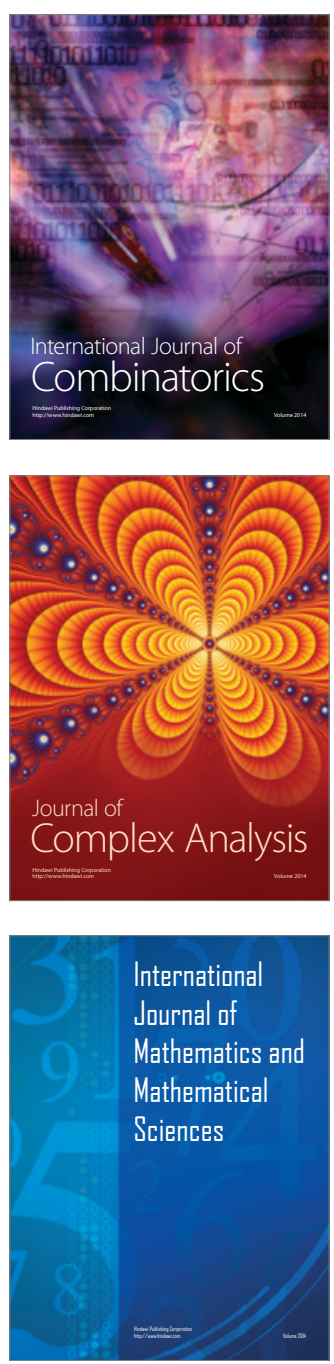
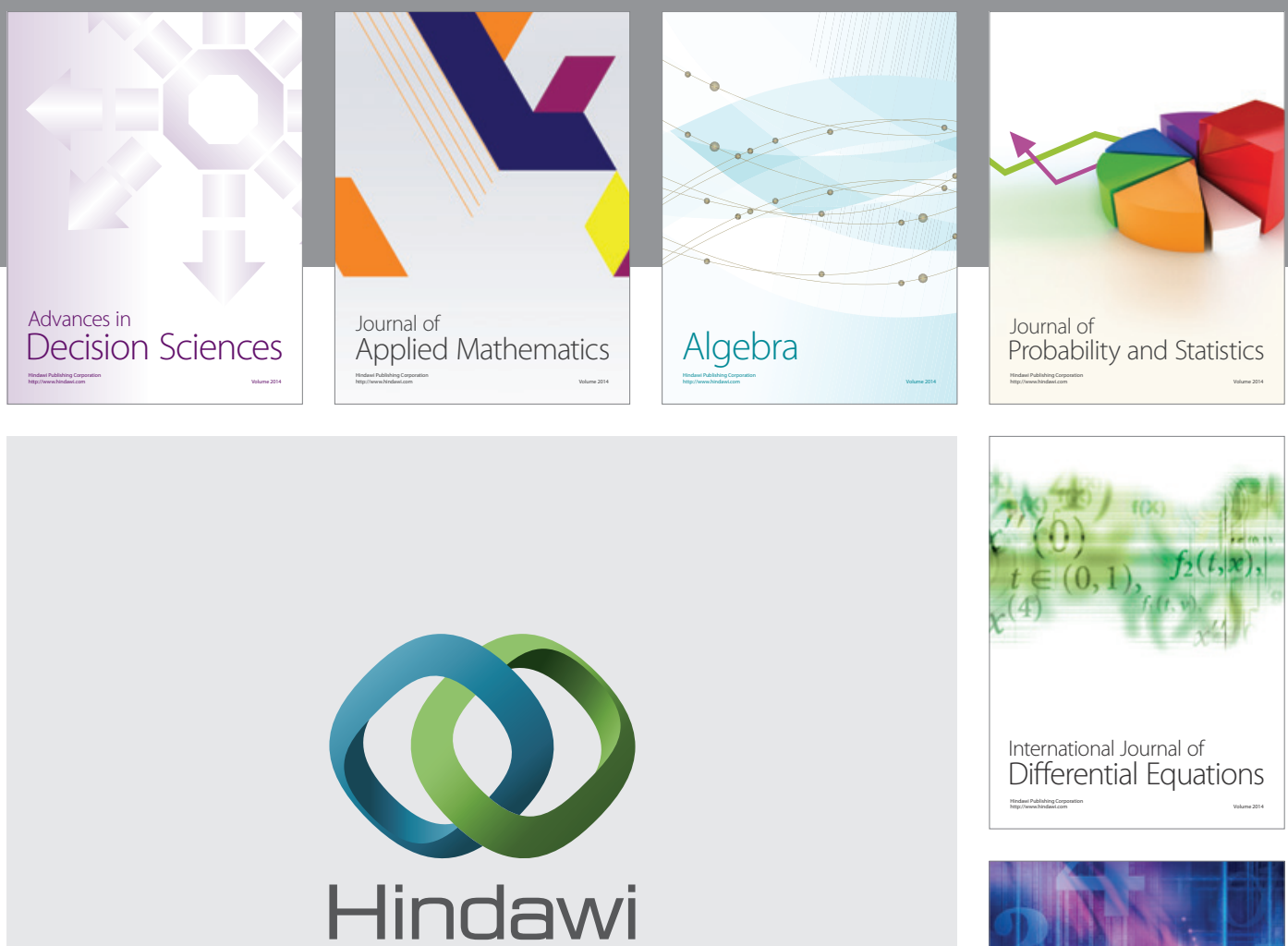

Submit your manuscripts at http://www.hindawi.com
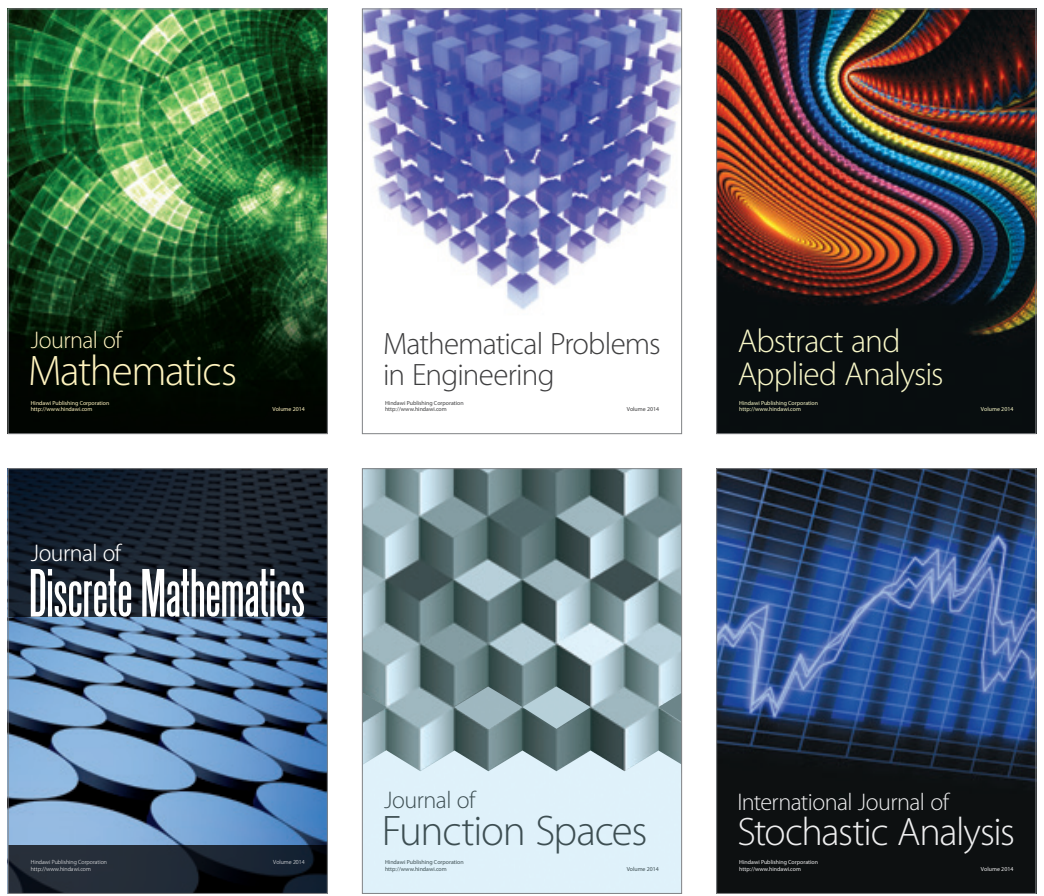

Journal of

Function Spaces

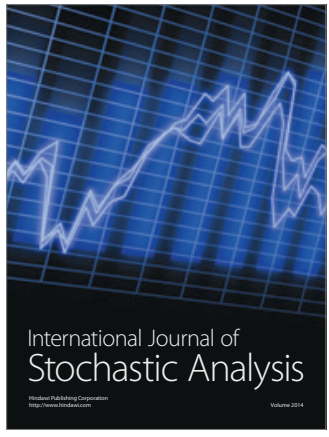

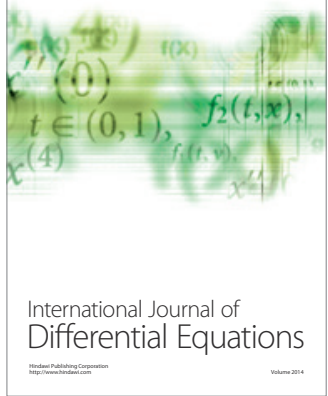
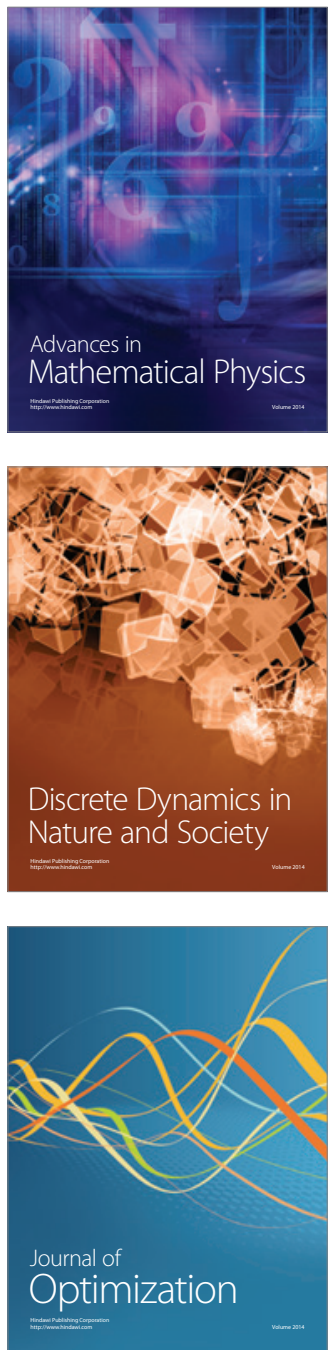